\title{
Emi1 protein accumulation implicates misregulation of the anaphase promoting complex/cyclosome pathway in ovarian clear cell carcinoma
}

\author{
Ines Gütgemann ${ }^{1, *}$, Norman L Lehman ${ }^{1}$, Peter K Jackson ${ }^{1,2}$ and Teri A Longacre ${ }^{1}$ \\ ${ }^{1}$ Department of Pathology, Stanford University, Stanford, CA, USA and ${ }^{2}$ Genentech Inc., South San \\ Francisco, CA, USA
}

\begin{abstract}
Clear cell carcinoma is a clinically and pathologically distinct entity among surface epithelial ovarian neoplasms, recognized for its resistance to standard platinum-based chemotherapy at advanced stage disease and poor prognosis. Despite advances in our understanding of the biology of other surface epithelial ovarian neoplasms, very little is known about the molecular genetic mechanisms that are involved in clear cell tumorigenesis. Early mitotic inhibitor-1 (Emi1) protein is a key cell cycle regulator, that promotes S-phase and mitotic entry by inhibiting the anaphase promoting complex. In cell culture systems, overexpression of Emi1 leads to tetraploidy and genomic instability, especially in the absence of normal p53 function. We investigated Emi1 protein expression in ovarian neoplasms using a tissue microarray constructed from 339 primary ovarian surface epithelial (serous, endometrioid, clear cell, and mucinous) and peritoneal (serous) neoplasms, stromal and mesenchymal tumors, germ cell tumors, and normal ovarian tissue. Significant overexpression of Emi1 protein was present in $82 \%(27 / 33)$ clear cell carcinoma, including one borderline tumor in a diffuse, granular cytoplasmic and perinuclear staining pattern, independent of patient age, presence of ovarian and/or pelvic endometriosis, and FIGO stage. In contrast, only 10\% (17/177) primary ovarian and primary peritoneal serous carcinomas, $0 \%(0 / 10)$ mucinous carcinomas, and 19\% (6/32) endometrioid carcinomas exhibited significant Emi1 protein overexpression. Accumulation of Emi1 protein was not linked to Ki-67 labeling index, but was directly correlated with cyclin E and inversely correlated with ER in clear cell carcinoma $(P<0.001)$. Emi1 protein expression was present in mixed endometrioid/clear cell tumors but absent in tumors with mixed serous/clear cell histology. These findings represent a potentially important insight into the molecular pathway underlying ovarian carcinogenesis and provide a possible cell cycle model for the development and progression of ovarian clear cell carcinoma.
\end{abstract}

Modern Pathology (2008) 21, 445-454; doi:10.1038/modpathol.3801022; published online 18 January 2008

Keywords: ovarian clear cell carcinoma; early mitotic inhibitor-1; Emi1; estrogen receptor; ovarian carcinoma

Ovarian clear cell carcinoma is a highly aggressive and often chemoresistant surface epithelial neoplasm associated with a poor 5 -year survival. ${ }^{1}$ Compared to the serous surface epithelial subtype, which comprises the majority of ovarian carcinomas, clear cell carcinoma is less common (less than 15\%) and is frequently associated with

Correspondence: Dr TA Longacre, MD, Department of Pathology, Stanford University School of Medicine, Room L235, 300 Pasteur Drive, Stanford, CA 94305, USA.

E-mail: longacre@stanford.edu

${ }^{*}$ Current address: Department of Pathology, University of Bonn, Bonn, Germany.

Received 09 July 2007; revised 15 October 2007; accepted 21 October 2007; published online 18 January 2008 ovarian and/or pelvic endometriosis, paraneoplastic hypercalcemia, and thromboembolic complications. Unlike its serous counterpart, clear cell carcinoma frequently presents at low FIGO stage and exhibits a comparatively poor response to conventional platinum-based chemotherapy at high-stage disease. The pathologic features of clear cell carcinoma are distinctive. Clear cell carcinoma, originally thought to be of mesonephric origin by Schiller (hence the old term 'mesonephroma'), is noted for its characteristic hobnail cells, clear cytoplasm, and mixed glandular, solid and tubulocystic architecture. In recent years, a dualistic model for the development and progression of ovarian cancer has been proposed, based in part on the presence or absence of recognizable precursor lesions. ${ }^{2}$ The evidence 
for this model is drawn primarily by comparing molecular genetic and gene expression data in serous borderline/low-grade carcinomas and their high-grade counterparts. Similar tumorigenic models for mucinous and endometrioid neoplasms have also been proposed. ${ }^{2}$ However, no such model exists for clear cell carcinoma and the pathobiology of this neoplasm continues to be poorly understood. ${ }^{3}$

Disruption of cell cycle checkpoints is a common underlying mechanism in human carcinogenesis leading to an increased rate of proliferation and/or decreased death of abnormal cells. ${ }^{4}$ In particular, misregulation of the p53 network is found in a significant proportion of human carcinomas, either linked to inactivating mutations of p53 or through alterations in genes whose products interact directly or indirectly with p53. ${ }^{5}$ p53 inactivating mutations, which occur commonly in serous ovarian cancer, are conspicuously absent in clear cell carcinoma, and although overexpression of MDM2, a negative regulator of the p53 protein may be operative in some of these tumors, the potential role of other key cell cycle regulators in clear cell tumorigenesis has not been well defined. ${ }^{6-9}$ Early mitotic inhibitor-1 (Emi1), a recently characterized E2F transcriptional target, is a key cell cycle regulator that is required for accumulation of mitotic cyclins and other critical cell cycle regulators during S and G2 phases. ${ }^{10-13}$ Emi1 functions as a pseudo-substrate inhibitor of the anaphase promoting complex/cyclosome (APC/ C). ${ }^{14-16}$ Upregulation of Emi1 mRNA has been found in a variety of malignant tumors compared to matched normal and benign tumor tissue. Indeed, Emi1 overexpression causes mitotic catastrophe and genomic instability through APC/C misregulation, and thus potentially contributes to tumorigenesis. ${ }^{10}$ In a large-scale screen of a variety of human neoplasms examining protein expression of Emi1 and tumorigenic APC/C substrates, overexpression of Emi1 was specifically implicated in clear cell carcinoma. ${ }^{17}$ To further evaluate the possible role of Emi1-mediated cell cycle misregulation in clear cell carcinoma, we examined the expression of Emi1 protein in a series of benign, borderline and malignant ovarian epithelial tumors and normal ovarian tissues using a tissue microarray platform and selected whole tissue sections. Our results support a potential link between Emi1 overexpression and tumorigenesis in clear cell carcinoma.

\section{Materials and methods}

\section{Tissue Selection and Tissue Microarray Construction}

Tissue microarrays were constructed from paraffinembedded tissue of primary ovarian and extra-ovarian tumors $(n=339)$ as well as normal ovarian tissue using a manual tissue arrayer (Beecher Instruments, Silver Spring, MD, USA) as described previously. ${ }^{18}$ These arrays contained
254 serous epithelial neoplasms (144 primary ovarian serous carcinomas, 13 recurrences of serous carcinomas, 38 peritoneal serous carcinomas, and 59 serous tumors of low malignant potential), 34 clear cell neoplasms (32 clear cell carcinomas, 1 borderline clear cell tumor, and 1 recurrence), 37 endometrioid neoplasms (32 endometrioid carcinomas and 5 endometrioid tumors of low malignant potential), and 31 mucinous epithelial tumors (10 mucinous carcinomas, 16 mucinous tumors of low malignant potential and 5 benign cystadenomas) as well as 10 normal ovaries, 3 teratomas, 1 mixed germ cell tumor, 1 yolk sac tumor, and 15 sex cordstromal tumors. Duplicate $0.6 \mathrm{~mm}$ cores were obtained for each case. Tumor tissue was obtained through the Surgical Pathology archives of the Department of Pathology at Stanford University under Institutional Review Board approval (protocol ID: 79607). All tumor cases were reviewed by one of the investigators (TAL) and classified by predominant histological type according to the WHO criteria. ${ }^{19}$ Primary vs recurrent tumors and tumors with mixed histology were noted. In addition, representative tissue sections of selected ovarian tumors as well as normal ovarian tissue from premenopausal and postmenopausal women with no history of ovarian cancer $(n=5)$ were obtained from the archives. The premenopausal ovary tissues were selected to represent follicular, luteal and post-luteal phases.

\section{Immunohistochemistry}

Serial sections of $4 \mu \mathrm{m}$ each were cut from the array blocks and deparaffinized by routine techniques. Each TMA was placed in $200 \mathrm{ml}$ of target retrieval solution, pH 6.0 (Envision Plus Detection Kit, Dako) for $20 \mathrm{~min}$ at $100^{\circ} \mathrm{C}$. After cooling for $20 \mathrm{~min}$, slides were quenched with $3 \% \mathrm{H}_{2} \mathrm{O}_{2}$ for $5 \mathrm{~min}$ before incubating with primary antibody using the Dako Autostainer. The primary antibodies used were as follows: affinity-purified rabbit anti-human-Emi1 polyclonal antibody (1:500 dilution); ${ }^{10}$ anti-estrogen receptor monoclonal antibody (clone 15d, 1:50 dilution, Dako); anti-cyclin E (clone 13A3, 1:40 dilution, Novocastra), and anti-Ki-67 (clone IVAK-2, 1:100 dilution, Dako). Labeling was performed with the Dako EnVision + System according to the manufacturer's protocol. Positive controls were included for each antibody. Negative controls were performed by replacing primary antibody with rabbit control serum. Specificity of Emi1 antibodies was previously demonstrated through prior incubation of the antiserum with purified Emi1 protein. ${ }^{17}$

Two of the investigators (IG and TAL) scored epithelial cytoplasmic and/or nuclear staining for Emi1 using a three-tiered scale, although only cytoplasmic staining was considered positive. Because Emi1 expression exhibited a variable pattern of staining intensity with less variation in overall 
percentage of positive cells, scoring was based on intensity of staining. Immunostaining in $<5 \%$ of tumor cells was considered negative and immunostaining in $>5 \%$ tumor cells was further scored as low level or high level, depending on the intensity of expression. Immunohistochemical staining for ER, cyclin E, and Ki-67, all of which exhibited uniform intensity of expression from case to case, was scored as negative $(<5 \%$ of cell nuclei staining positively), weakly positive (5-30\% of nuclei positive), or strongly positive ( $>30 \%$ nuclei positive). Only appropriate subcellular localization of detectable signal was considered for assessment of percentage staining (cytoplasmic expression for Emi1 and nuclear expression for ER, Ki-67, and cyclin E). Cases in which immunoreactivity could not be assessed for technical reasons (eg, failure of the tissue cores to stick to the slide or no representative tissue in either core) were excluded from analysis. In cases where there was a discrepancy in the staining result recorded for the duplicate cores, the higher score was accepted for that case (eg, for a case with negative staining in one core and low-level staining in the other, the case was considered to show low level expression).

\section{FIGO Stage and Follow-Up}

Patient age, FIGO stage of disease, and clinical follow-up were obtained for all tumor cases by review of the hospital charts.

\section{Statistical Analysis}

Statistical analysis was performed using Microstat statistical software (Ecosoft, Indianapolis, IN, USA). Fisher's exact test, and where indicated, the trend test, a modification of the $\chi^{2}$ test for ordered categorical data, was performed. The two-sided level of significance was set at $P<0.05$. Similarly, $95 \%$ confidence intervals and the Spearman's correlation coefficient were calculated.

\section{Results}

\section{Emi1 Protein Expression in Ovarian Neoplasms}

To understand the role of Emi1 protein in ovarian tumors, we used immunohistochemical analysis to examine Emi1 protein in normal ovary and ovarian tumors. Normal ovarian stromal tissue demonstrated no significant Emi1 expression, except in tissues containing post-antral, involuting or recently ruptured follicles. The granulosa cell layer of these Graafian follicles (Figure 1) and the ovarian cortical stromal cells near areas of prior follicular rupture or involution (Figure 2) demonstrated discrete cytoplasmic Emi1 expression (Figure 2). Emi1 expression by surface epithelium or (when present) epithelial inclusion cysts or clefts was either absent

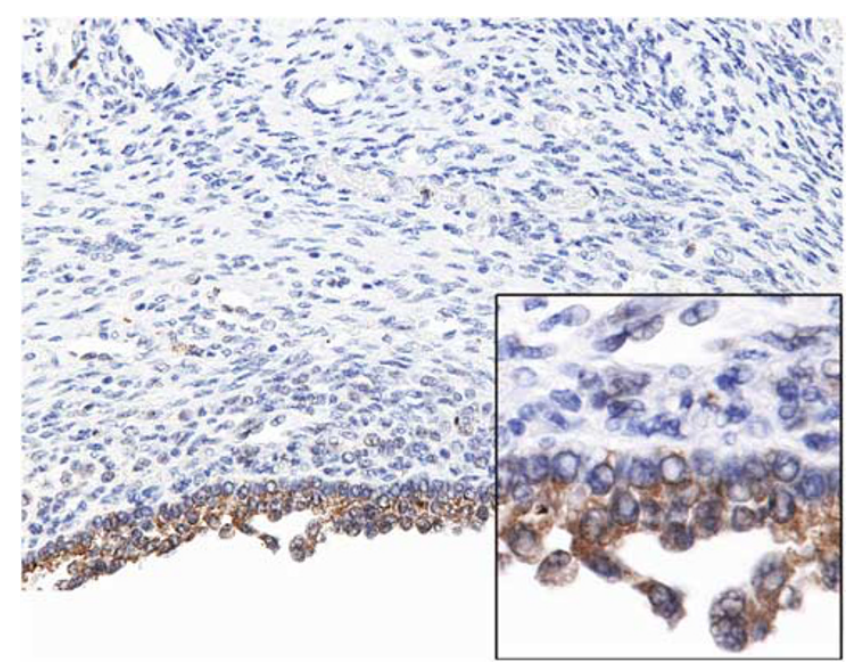

Figure 1 Emi1 expression in normal ovary. Granulosa cells in Graafian follicles express high level Emi1 in a cytoplasmic distribution (inset), whereas low level expression is present in scattered thecal cells, but not in surrounding cortical stromal tissue.

or weakly positive (scored as low level expression, Figure 2). Fallopian tube epithelium exhibited similar, but more extensive expression of Emi1 (Figure 3).

In contrast, low- or high-level Emi1 expression was present in a significant proportion of ovarian surface epithelial tumors (43\%). Emi1 expression in these tumors exhibited a diffuse, cytoplasmic and perinuclear granular staining pattern that was particularly prominent in the clear cell tumors (Figure 4), whereas the majority of serous, endometrioid, and mucinous neoplasms either exhibited no expression or only low levels of Emi1 expression (Table 1). Clear cell tumors exhibited high-level Emi1 expression (Figure 5) in 27 of 33 or $82 \%$ of clear cell carcinoma (including one borderline tumor), whereas high-level Emi1 protein expression was present only in $10 \%(17 / 177)$ primary ovarian and primary peritoneal serous carcinomas, 0/10 mucinous carcinomas, and $19 \%(6 / 32)$ endometrioid carcinomas. High-level Emi1 expression was more common in serous tumors of low malignant potential and low-grade serous carcinomas than in the high-grade serous cancers (17 vs $10 \%)$. Three of the six endometrioid cancers (all grade 2, two high stage) with high Emi1 expression were associated with endometriosis, one with mixed endometrioid and clear cell histology. Emi1 overexpression was also seen in $1 / 5$ endometrioid tumors of low malignant potential and $3 / 4$ endometrioisis unassociated with carcinoma, two of which were atypical (Figure 6). ${ }^{20,21}$ Two germ cell tumors (one yolk sac tumor and one dysgerminoma) and one (of six) adult granulosa cell tumor demonstrated strong Emi1 expression; all other sex cord neoplasms studied were negative for Emi1. Altogether, taking low- and high-level expression, 


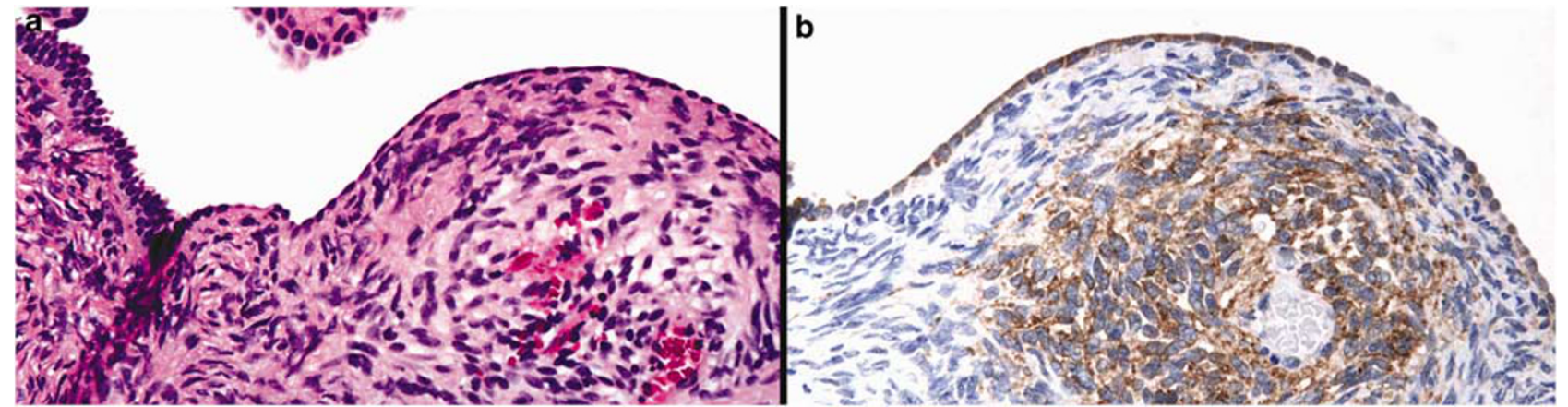

Figure 2 Emi1 expression in normal ovary. (a) Cortical surface near site of prior follicle rupture (H\&E). (b) Discrete Emi1 expression in surrounding stroma near site of ruptured follicle and in overlying surface epithelium.

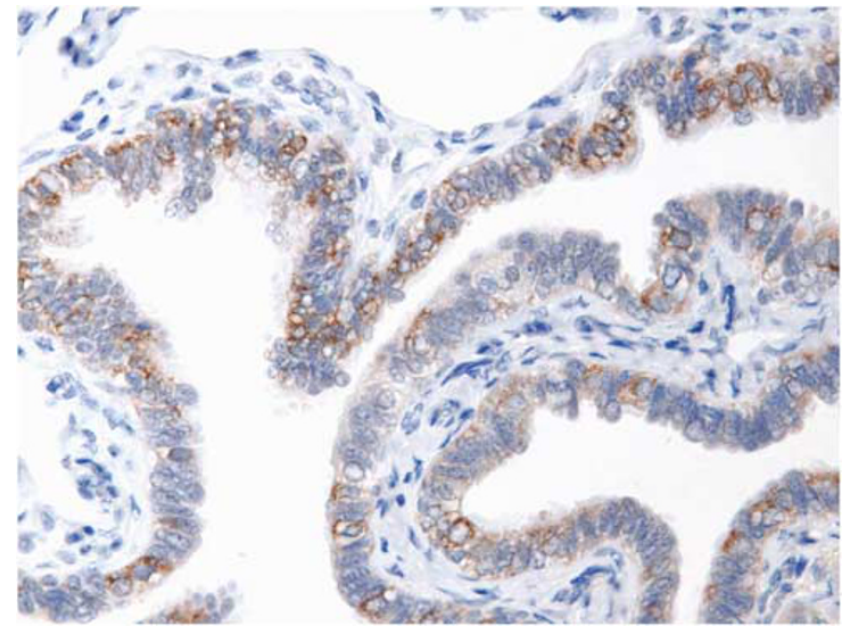

Figure 3 Emi1 expression in normal fallopian tube. The intercalated epithelium (so-called peg cells) preferentially expresses Emi1.

clear cell carcinoma expressed Emi1 in 85\% (30/33) of cases. The strong expression of Emi1 in clear cell carcinomas was highly significant $(P<0.0001,95 \%$ confidence interval $[65 \%, 93 \%])$. Of the three clear cell carcinomas with no Emi1 expression, one was classified as mixed clear cell and serous carcinoma and one was a recurrence at 6 months (initial clear cell carcinoma with high Emi1 expression). No statistically significant differences in Emi1 expression occurred among the remaining groups after removal of the clear cell group from the analysis $(P=0.29)$.

\section{Emi1 Protein Accumulation in Relation to ER Expression in Clear Cell Carcinoma}

Since the loss of estrogen receptor in breast and ovarian tumors may contribute to increased and unregulated expression of cell cycle regulators, we also evaluated the link between ER and Emi1 in ovarian tumors. Emi1 expression was inversely correlated with ER protein expression in clear cell carcinoma (Figure 4), with only 3/33 clear cell carcinomas exhibiting low levels of ER expression (two of the three were Emi1-negative, of which one was mixed serous and clear cell carcinoma). In contrast, more than half of the serous carcinomas (91/177; $52 \%), 52 \%$ of endometrioid carcinomas, $10 \%$ of mucinous carcinomas and $77 \%$ of serous tumors of low malignant potential expressed the ER protein (Table 1). Comparison between all five groups was statistically significant $(P<0.001)$. Thus, ER-negative cases were most frequent among clear cell carcinomas and mucinous carcinomas and ERpositive cases were most frequent among the serous tumors of low malignant potential. No statistically significant correlation between Emi1 and ER expression was found in any group but clear cell carcinoma, where an inverse correlation of Emi1 and ER expression was demonstrated (Spearman's coefficient $-0.454, P=0.008$ ).

\section{Emi1 Protein Accumulation in Relation to Cyclin E and Ki-67 Labeling Index in Clear Cell Carcinoma}

In addition to ER status, cyclin E levels have been clearly implicated in the prognosis of breast cancer ${ }^{22}$ and potentially, ovarian cancer. ${ }^{23,24}$ To test this link, we evaluated the relationship between expression of cyclin E and Emi1 in ovarian tumors. High-cyclin E expression was present in 29/33 (88\%) CCC, $64 \%$ serous carcinomas, $26 \%$ endometrioid carcinomas and $0 \%$ mucinous carcinomas. Pairwise comparison between the histologic subtypes showed significant overexpression of cyclin E in the clear cell carcinoma group compared to the other subtypes (Table 1), and this was directly correlated with Emi1 overexpression in clear cell carcinoma $(P<0.0001)$. Overexpression of Emi1 occurred in association with elevated cyclin $\mathrm{E}$ in all but one clear cell carcinoma (Figure 4).

To determine whether Emi1 expression in the tumor tissue was reflective of a generalized increase in tumor cell proliferation, Emi1 expression was 


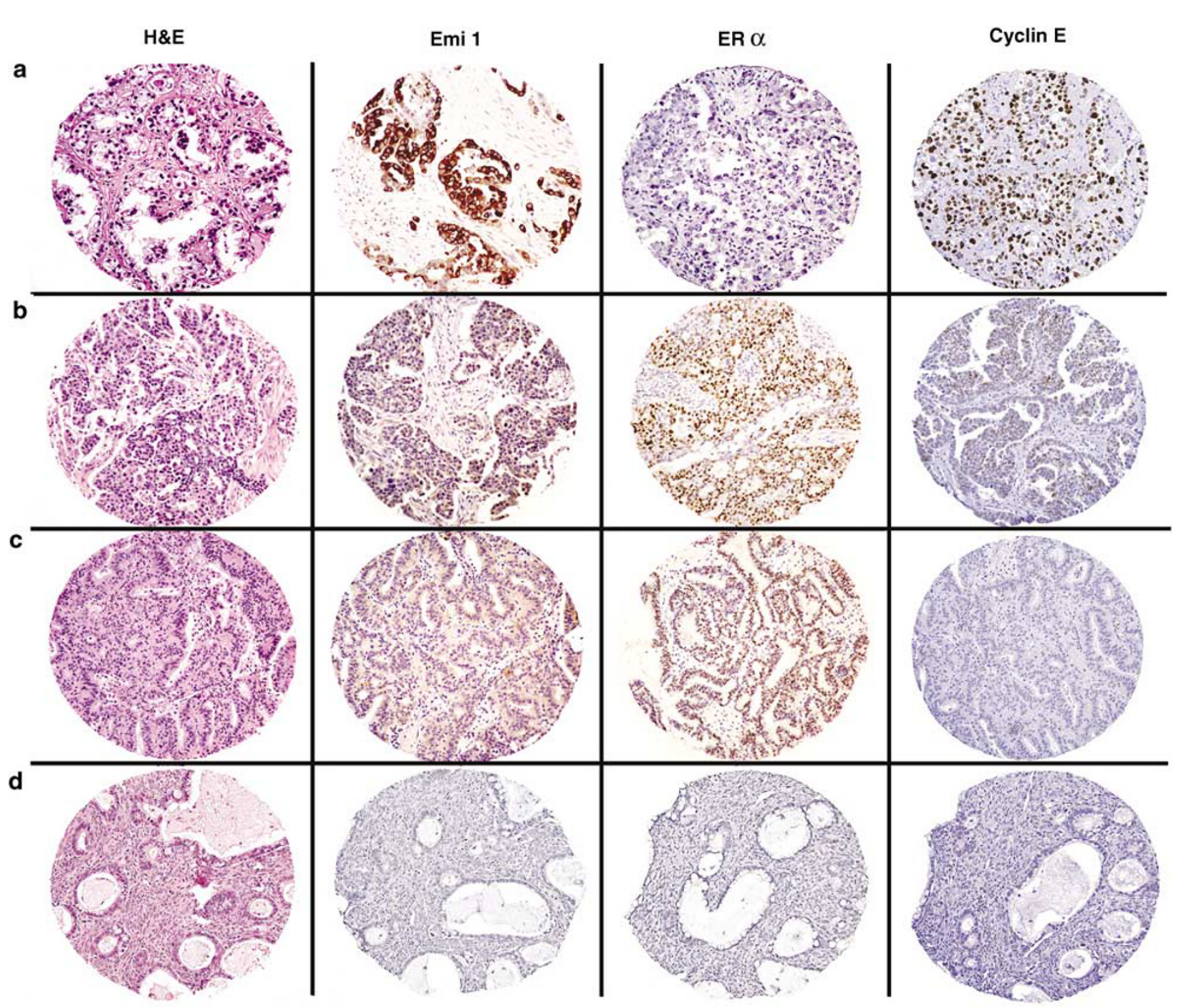

Figure 4 Differential expression of Emi1, ER, and cyclin E in ovarian surface epithelial neoplasms: (a) clear cell carcinoma, (b) serous carcinoma, (c) endometrioid carcinoma, and (d) mucinous carcinoma. Immunohistochemical detection of Emi1, ER, and cyclin E are depicted. Panels from left to right: H\&E, anti-Emi1, anti-ER, and anti-cyclin E.

Table 1 Expression of Emi1, ER, and cyclin E in ovarian surface epithelial carcinomas

\begin{tabular}{lccc}
\hline & $\begin{array}{c}\text { Emi1 } \\
(\%)\end{array}$ & $\begin{array}{c}E R \\
(\%)\end{array}$ & $\begin{array}{c}\text { Cyclin } E \\
(\%)\end{array}$ \\
\hline CCC & $27 / 33(82)^{\mathrm{a}}$ & $3 / 33(10)$ & $29 / 33(88)$ \\
Serous & $17 / 177(9)$ & $91 / 177(51)$ & $118 / 177(67)$ \\
Endometrioid & $6 / 32(19)$ & $17 / 32(52)$ & $8 / 32(25)$ \\
Mucinous & $0 / 10(0)$ & $1 / 10(10)$ & $0 / 10(0)$ \\
$P$-value & $<0.0001$ & $<0.0001$ & $<0.0001$ \\
\hline
\end{tabular}

CCC, clear cell carcinoma.

Values indicate cases showing positive staining per total number of cases examined per subgroup. Number in parenthesis indicates \% of tumors expressing the marker per subgroup.

${ }^{\mathrm{a}}$ Includes one borderline clear cell tumor.

${ }^{*} P$-value reflects expression pattern in CCC compared to other histologic types (both individually and in aggregate). correlated with Ki-67 labeling in the tissue arrays and in representative full tissue sections. Although Ki-67 expression was highly variable among the tumors, levels of expression were generally lower in the clear cell carcinomas than in all serous carcinomas combined and no correlation between Emi1 expression and clear cell carcinoma tumor cell proliferation, as assessed by either Ki-67 labeling or mitotic index was found (data not shown).

\section{Emi1 Expression in Putative Precursor Lesions and in Early and Late Stage Clear Cell Carcinoma}

Emi1 expression was absent in 4/4 examples of assessable ovarian endometriosis, while $2 / 2$ 

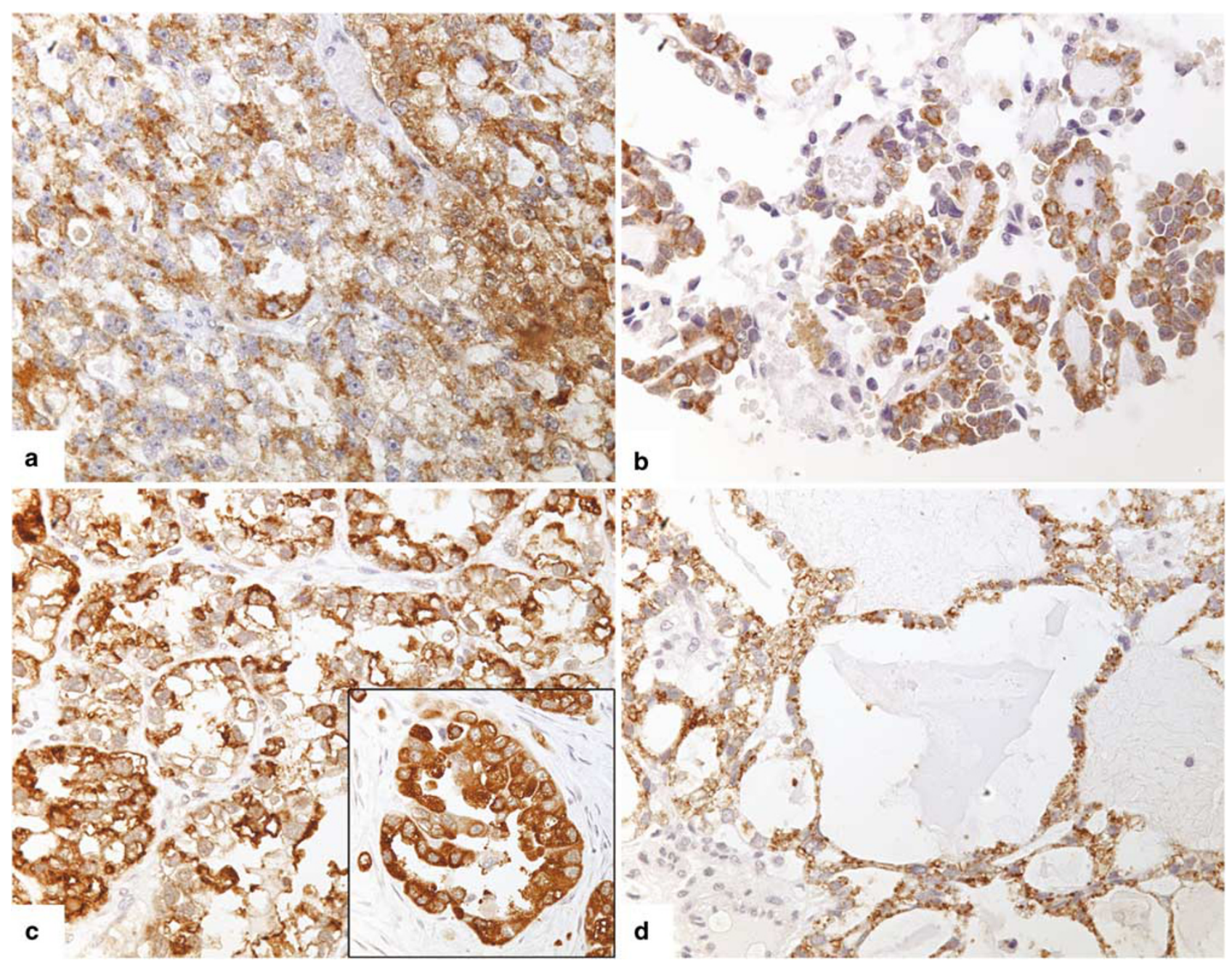

Figure 5 Emi1 expression in clear cell carcinoma With (a) solid, (b) papillary, (c) glandular, and (d) macrocystic patterns. Expression is predominantly cytoplasmic (inset, c).

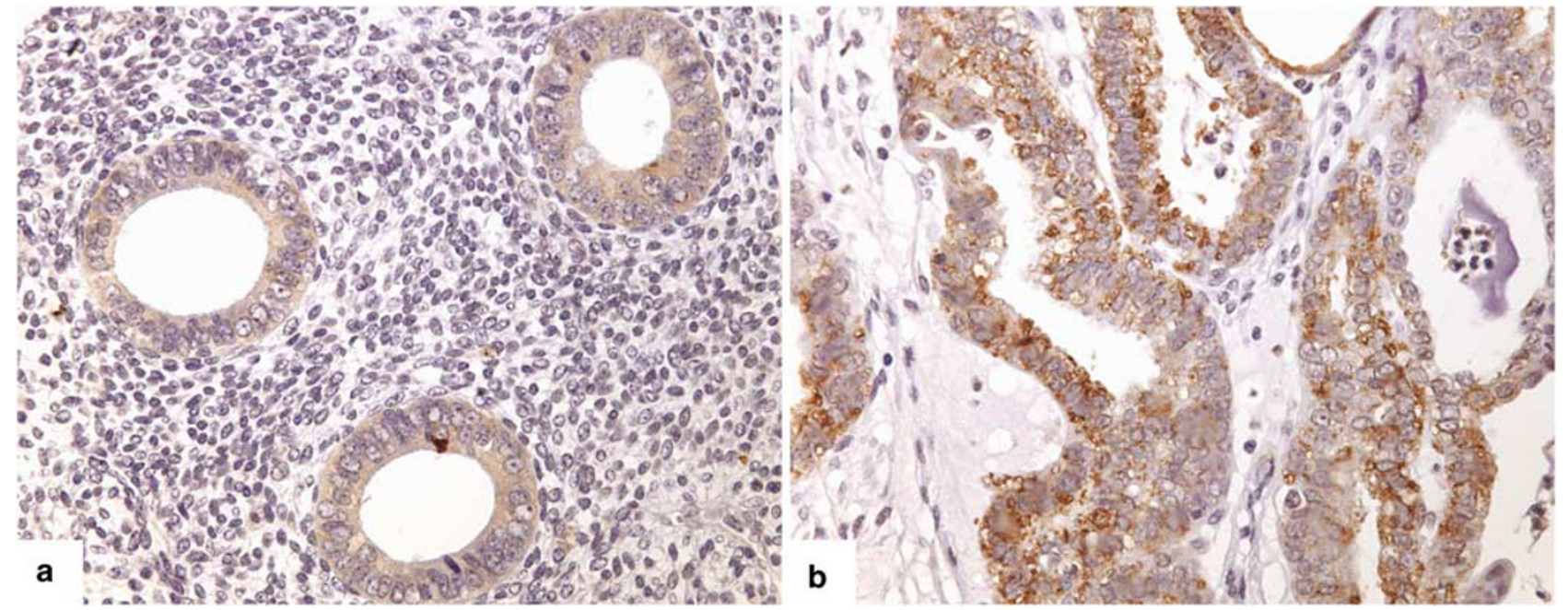

Figure 6 Emi1 expression in (a) endometriosis (low level) and (b) atypical endometriosis (high level).

examples of atypical ovarian endometriosis expressed Emi1 (one low level and one high level). Twelve clear cell carcinomas were associated with ovarian and/or pelvic endometriosis; each of these tumors expressed Emi1. Both clear cell carcinomas associated with a well-defined adenofibromatous 
component also expressed Emi, as did the single borderline clear cell tumor.

The 33 clear cell carcinomas represented 32 primary tumors and 1 recurrence. Primary clear cell carcinoma was equally distributed among low-stage and high-stage disease: 11 FIGO IA, 3 FIGO II, 16 FIGO III, and 2 FIGO IV. Twenty-one (64\%) were unilateral, seven were associated with surface metastases to the contralateral ovary (21\%) and five were bilateral with ovarian parenchymal involvement $(15 \%)$. Of the cases with bilateral involvement, two exhibited mixed serous and clear cell histology. All FIGO stage I and II clear cell carcinomas expressed Emi1, whether or not they were associated with endometriosis or an adenofibromatous component. Complete absence of Emi1 expression in clear cell carcinomas occurred only in FIGO stage III (one case: pure clear cell carcinoma, ER + ), FIGO stage IV (one case: mixed serous and clear cell carcinoma, $\mathrm{ER}+$ ), and recurrent (one case: pure clear cell carcinoma, ER-) disease. Emi1- clear cell carcinomas exhibited clinically aggressive disease with early recurrence (2/2 with available follow-up vs 7/16 $\mathrm{Emi}+$ with available follow-up), but the numbers of cases were too few to draw statistical inferences.

\section{Discussion}

Clear cell carcinoma is a clinically and pathologically distinct entity among surface epithelial ovarian neoplasms, recognized for resistance to standard platinum-based chemotherapy at advanced stage disease and poor prognosis. The accumulated evidence from a variety of disciplines indicates that clear cell carcinoma also has several distinct cytogenetic, molecular genetic, and gene expression alterations when compared to other more common surface epithelial neoplasms..$^{8,25-28}$ Not surprisingly, some of the differentially expressed genes found on mRNA expression studies of ovarian carcinoma fall into a proliferation cluster or cluster with genes encoding proteins involved in cell cycle progression. ${ }^{26}$ Misregulation of the G1/S transition in the cell cycle is a fundamental component of the cellular transformation process and G1/S regulatory defects have been reported in a variety of human malignancies.

Here, we studied the expression of Emi1, a recently described key cell cycle regulator that is essential for normal regulation of the $\mathrm{APC} / \mathrm{C}$, in a series of normal, benign, and malignant ovarian tissues using a tissue microarray platform. We found that Emi1 protein, although expressed in a variety of malignant ovarian tumors, was significantly overexpressed in clear cell carcinomas with 85\% exhibiting at least some degree of Emi1 protein expression, and most at high levels (82\% of all clear cell carcinomas). Emi1 protein accumulation in clear cell carcinoma was directly associated with upregulation of cyclin $\mathrm{E}$ and, with one exception, was found only when cyclin E was overexpressed, but was not related to a generalized increased proliferative activity. The next highest frequency of Emi1 overexpression in the ovarian surface epithelial tumor subsets occurred in endometrioid carcinomas (19\%). We also found low levels of Emi1 expression in two cases of atypical endometriosis, and in borderline tumors of each subtype. Taken together, these findings provide strong circumstantial evidence for the role of Emi1-mediated misregulation of the $\mathrm{APC} / \mathrm{C}$ pathway in clear cell carcinoma. Moreover, misregulation of the pathway is likely to be an early event, because complete absence of Emi1 in clear cell carcinoma appears to occur only in high stage or recurrent disease. Misregulation of the APC/C pathway has also been reported in colon and breast cancers and although APC/C substrates have been implicated in ovarian carcinogenesis, specific upregulation of a key cell cycle regulator of the APC/C in clear cell carcinoma has not been previously documented. ${ }^{17,26,29-32}$

Upregulation of Emi1, which also occurs at the transcriptional level, has been reported in other neoplasms in association with cyclin E overexpression. ${ }^{10,17}$ Coexpression of these two proteins is consistent with Emi1 being an E2F target, since cyclin E/cdk2 phosphorylates pRb and promotes the transcription of E2F target genes. ${ }^{10}$ Overexpression of cyclin-dependent kinase or cyclin $\mathrm{E}$ may lead to activated E2F and overexpression of the E2Fdependent APC/C regulator Emi1. ${ }^{10}$ Alternatively, loss of upstream regulators such as $\mathrm{pRb}$ or cdk inhibitors with resultant misregulation and accumulation of Emi1 protein may account for the observed protein overexpression in this study. In clear cell carcinoma, overexpression of Emi1 may contribute to aggressive tumor growth by driving cell cycle progression at an increased rate and/or inducing genomic instability. ${ }^{33}$ Tetraploidy, an important intermediary step in tumor progression, has been reported in a significant proportion of ovarian clear cell carcinoma and mitotic errors with centrosome overduplication and abnormal spindle formation have been linked to Emi1 accumulation in the absence of normal p53 function. ${ }^{7,12,33-35}$ In this model, additional p53 pathway destabilizing mechanisms, such as MDM2 alterations, would presumably occur, since p53 mutations are unusual in clear cell carcinoma. ${ }^{10,36}$

Recently, Shih and Kurman ${ }^{2}$ have proposed a dualistic tumor progression model for ovarian carcinogenesis, based on the presence or absence of precursor lesions. Type I tumors, represented by low-grade serous carcinoma, mucinous carcinoma, endometrioid carcinoma, and clear cell carcinoma arise in a stepwise manner from well-recognized precursor lesions, whereas Type II tumors appear to arise de novo from surface epithelium or inclusions. ${ }^{37}$ The development of ovarian endometrioid and clear cell neoplasia in association with endometriosis is well established and models of 


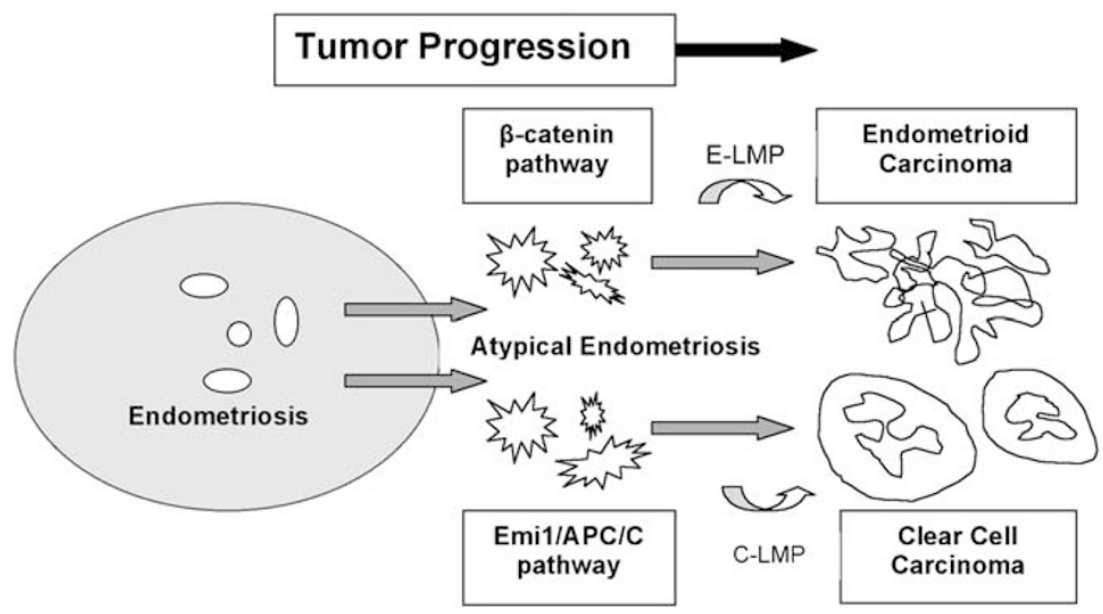

Figure 7 Proposed model for clear cell tumorigenesis. Endometriosis is considered to be the precursor lesion of clear cell and endometrioid carcinoma, possibly via intermediary atypical endometriosis. Subsequent steps in endometrioid tumorigenesis involve the $\mathrm{Wnt} / \beta$-catenin pathway, whereas the Emi1/APC/C pathway is active in clear cell tumorigenesis. Both tumors may proceed through a borderline or low malignant potential lesion (E-LMP or C-LMP, respectively), but are more likely to arise directly from endometriosis/ atypical endometriosis in this model.

tumorigenesis involving acquisition of a series of genetic alterations in foci of endometriosis have been proposed..$^{2,6,30,38-40}$ However, the pathways for the development of clear cell carcinoma and endometrioid carcinoma differ in several important respects. First, tumors with predominantly endometrioid differentiation tend to retain ER whereas those with predominantly clear cell differentiation lose $\mathrm{ER}$, analogous to poor prognosis ER-negative breast cancers. Second, alterations in the $\mathrm{Wnt} / \beta$-catenin signaling pathway have been consistently identified in ovarian endometrioid tumors with and without endometriosis, but no such alterations have been consistently identified in clear cell carcinoma. ${ }^{41-43}$ Third, the proliferative index of endometrioid tumors is significantly higher than in clear cell carcinoma, even though the latter tumor tends to behave in a more aggressive fashion when high stage. $^{25,30,44}$ Our data, combined with those of others, provide strong evidence for the role of Emi1mediated misregulation of the APC/C pathway in ovarian clear cell carcinoma and provide, for the first time, a tenable tumor progression model for these tumors. This tumor progression model, based on Emi1-driven misregulation of the APC/C pathway, does not exclude the role of other molecular defects that have been identified in ovarian clear cell carcinoma, and likely acts in synergy with some of these other key events to enhance tumor formation (Figure 7). ${ }^{45}$

Although not specifically examined in this study, Emi1 also appears to be overexpressed in some ovarian germ cell and stromal tumors. In this series, dysgerminoma and yolk sac tumors exhibited high levels of Emi1 expression, whereas all immature and mature teratomas showed no Emi1 expression. Emi1 and/or structurally similar Emi1-related proteins are considered to be integral to the maintenance of oocyte arrest at metaphase of meiosis II by their actions on the APC/C complex. ${ }^{11}$ In the normal ovary, Emi1 is overexpressed at low levels in ovarian granulosa cells in post-antral follicles and in the ovarian stroma during follicle rupture and atresia, but not in the oocytes or stroma of primordial follicles (Figure 1). The relationship, if any between Emi1 during normal follicular development and its overexpression in germ cell tumors, such as yolk sac tumors (and rarely, granulosa cell tumors) as well as in germinomas ${ }^{17}$ is unclear and the numbers of cases studied are too small to draw statistical inferences, but possible activation of the APC/C pathway in these tumors deserves further study.

In summary, ovarian clear cell carcinoma exhibits high-level Emi1 protein overexpression in the vast majority of clear cell carcinoma and appears to be lost only in recurrent and poor prognosis high-stage disease. Emi1 accumulation is associated with elevated cyclin $\mathrm{E}$ and is unrelated to proliferative activity, consistent with an Emi1-mediated misregulation of the APC/C pathway. ${ }^{33}$ The role of Emi1 and the APC/C pathway in clear cell carcinoma is supported by low-to-intermediate level Emi1 protein expression in atypical endometriosis and high expression in some endometrioid carcinomas, including tumors with mixed endometrioid and clear cell histology. These findings represent a potentially important insight into the molecular pathway underlying clear cell carcinogenesis and provide a possible novel cell cycle regulatory model for future studies aimed at elucidating the pathogenesis and mechanisms of chemoresistance of these neoplasms.

\section{Acknowledgements}

We thank JY Hsu MD, PhD for assistance in initial Emi1 antibody preparation, K Montgomery and 
M Madek for their technical assistance and C Nicolay (University of Bonn, Germany) for assistance with statistical analysis.

\section{References}

1 International Federation of Gynecology and Obstetrics. Annual report on the results of treatment in gynecological cancer. Int J Gynecol Obstet 1989;28:189-190.

2 Shih I-M, Kurman R. Ovarian tumorigenesis: a proposed model based on morphological and molecular genetic analysis. Am J Pathol 2004;164:1511-1518.

3 Gilks CB. Subclassification of ovarian surface epithelial tumors based on correlation of histologic and molecular pathologic data. Int J Gynecol Pathol 2004;23:200-205.

4 Johnson DG, Walker CL. Cyclins and cell cycle checkpoints. Annu Rev Pharmacol Toxicol 1999;39: 295-312.

5 Vogelstein B, Lane D, Levine AJ. Surfing the p53 network. Nature 2000;408:307-310.

6 Okuda T, Otsuka J, Sekizawa A, et al. p53 mutations and overexpression affect prognosis of ovarian endometrioid cancer but not clear cell cancer. Gynecol Oncol 2003;88:318-325.

7 Skirnisdottir I, Seidal T, Karlsson MG, et al. Clinical and biological characteristics of clear cell carcinomas of the ovary in FIGO stages I-II. Int J Oncol 2005;26:177-183.

8 Ho ES, Lai CR, Hsieh YT, et al. p53 mutation is infrequent in clear cell carcinoma of the ovary. Gynecol Oncol 2001;80:189-193.

9 Skomedal H, Kristensen GB, Abeler VM, et al. TP53 protein accumulation and gene mutation in relation to overexpression of MDM2 protein in ovarian borderline tumours and stage I carcinomas. J Pathol 1997;181: 158-165.

$10 \mathrm{Hsu}$ JY, Reimann JD, Sorensen CS, et al. E2Fdependent accumulation of hEmi1 regulates $S$ phase entry by inhibiting APC(Cdh1). Nat Cell Biol 2002;4:358-366.

11 Reimann JD, Jackson PK. Emi1 is required for cytostatic factor arrest in vertebrate eggs. Nature 2002;416:850-854.

12 Margottin-Goguet F, Hsu JY, Loktev A, et al. Prophase destruction of Emi1 by the SCF(betaTrCP/Slimb) ubiquitin ligase activates the anaphase promoting complex to allow progression beyond prometaphase. Dev Cell 2003;4:813-826.

13 Guardavaccaro D, Kudo Y, Boulaire J, et al. Control of meiotic and mitotic progression by the $\mathrm{F}$ box protein beta-Trcp1 in vivo. Dev Cell 2003;4:799-812.

14 Miller JJ, Summers MK, Hansen DV, et al. Emi1 stably binds and inhibits the anaphase-promoting complex/ cyclosome as a pseudosubstrate inhibitor. Genes Dev 2006;20:2410-2420.

15 Reimann JD, Gardner BE, Margottin-Goguet F, et al. Emi1 regulates the anaphase-promoting complex by a different mechanism than Mad2 proteins. Genes Dev 2001;15:3278-3285.

16 Reimann JD, Freed E, Hsu JY, et al. Emi1 is a mitotic regulator that interacts with Cdc20 and inhibits the anaphase promoting complex. Cell 2001;105:645-655.

17 Lehman NL, Tibshirani R, Hsu JY, et al. Oncogenic regulators and substrates of the anaphase promoting complex/cyclosome are frequently overexpressed in malignant tumors. Am J Pathol 2007;170: 1793-1805.

18 Gilks CB, Vanderhyden BC, Zhu S, et al. Distinction between serous tumors of low malignant potential and serous carcinomas based on global mRNA expression profiling. Gynecol Oncol 2005;96:684-694.

19 Tavassoli FA, Devillee P. World Health Organization Classification of Tumours. Pathology and Genetics. Tumours of the Breast and Female Genital Organs. IARC Press: Lyon, 2003.

20 LaGrenade A, Silverberg SG. Ovarian tumors associated with atypical endometriosis. Hum Pathol 1988;19:1080-1084.

21 Czernobilsky B, Morris WJ. A histologic study of ovarian endometriosis with emphasis on hyperplastic and atypical changes. Obstet Gynecol 1979;53: 318-323.

22 Potemski P, Kusinska R, Watala C, et al. Cyclin E expression in breast cancer correlates with negative steroid receptor status, HER2 expression, tumor grade and proliferation. J Exp Clin Cancer Res 2006;25: 59-64

23 Eder AM, Sui X, Rosen DG, et al. Atypical PKCiota contributes to poor prognosis through loss of apicalbasal polarity and cyclin $\mathrm{E}$ overexpression in ovarian cancer. Proc Natl Acad Sci USA 2005;102: 12519-12524.

24 Rosen DG, Yang G, Deavers MT, et al. Cyclin E expression is correlated with tumor progression and predicts a poor prognosis in patients with ovarian carcinoma. Cancer 2006;106:1925-1932.

25 Tsuchiya A, Sakamoto M, Yasuda J, et al. Expression profiling in ovarian clear cell carcinoma: identification of hepatocyte nuclear factor-1 beta as a molecular marker and a possible molecular target for therapy of ovarian clear cell carcinoma. Am J Pathol 2003;163: 2503-2512.

26 Schaner ME, Ross DT, Ciaravino G, et al. Gene expression patterns in ovarian carcinomas. Mol Biol Cell 2003;14:4376-4386.

27 Schwartz DR, Kardia SL, Shedden KA, et al. Gene expression in ovarian cancer reflects both morphology and biological behavior, distinguishing clear cell from other poor-prognosis ovarian carcinomas. Cancer Res 2002;62:4722-4729.

28 Dent J, Hall GD, Wilkinson N, et al. Cytogenetic alterations in ovarian clear cell carcinoma detected by comparative genomic hybridisation. $\mathrm{Br} \mathrm{J}$ Cancer 2003;88:1578-1583.

29 Wang Q, Moyret-Lalle C, Couzon F, et al. Alterations of anaphase-promoting complex genes in human colon cancer cells. Oncogene 2003;22:1486-1490.

30 Sato N, Tsunoda H, Nishida $M$, et al. Loss of heterozygosity on 10q23.3 and mutation of the tumor suppressor gene PTEN in benign endometrial cyst of the ovary: possible sequence progression from benign endometrial cyst to endometrioid carcinoma and clear cell carcinoma of the ovary. Cancer Res 2000;60: 7052-7056.

31 Park KH, Choi SE, Eom M, et al. Downregulation of the anaphase-promoting complex (APC)7 in invasive ductal carcinomas of the breast and its clinicopathologic relationships. Breast Cancer Res 2005;7: R238-R247.

32 Shapira M, Kakiashvili E, Rosenberg $\mathrm{T}$, et al. The mTOR inhibitor rapamycin down-regulates the 
expression of the ubiquitin ligase subunit Skp2 in breast cancer cells. Breast Cancer Res 2006;8:R46.

33 Lehman NL, Verschuren EW, Hsu JY, et al. Overexpression of the anaphase promoting complex/ cyclosome inhibitor Emi1 leads to tetraploidy and genomic instability of p53-deficient cells. Cell Cycle 2006;5:1569-1573.

34 Hernando E, Nahle Z, Juan G, et al. Rb inactivation promotes genomic instability by uncoupling cell cycle progression from mitotic control. Nature 2004;430: 797-802.

35 Borel F, Lohez OD, Lacroix FB, et al. Multiple centrosomes arise from tetraploidy checkpoint failure and mitotic centrosome clusters in p53 and RB pocket protein-compromised cells. Proc Natl Acad Sci USA 2002;99:9819-9824.

36 Shimizu M, Nikaido T, Toki T, et al. Clear cell carcinoma has an expression pattern of cell cycle regulatory molecules that is unique among ovarian adenocarcinomas. Cancer 1999;85:669-677.

37 Shih Ie M, Kurman RJ. Molecular pathogenesis of ovarian borderline tumors: new insights and old challenges. Clin Cancer Res 2005;11:7273-7279.

38 Modesitt SC, Tortolero-Luna G, Robinson JB, et al. Ovarian and extraovarian endometriosis-associated cancer. Obstet Gynecol 2002;100:788-795.
39 Thomas EJ, Campbell IG. Molecular genetic defects in endometriosis. Gynecol Obstet Invest 2000; 50(Suppl 1):44-50.

40 Fukunaga M, Nomura K, Ishikawa E, et al. Ovarian atypical endometriosis: its close association with malignant epithelial tumours. Histopathology 1997;30: 249-255.

41 Catasus L, Bussaglia E, Rodrguez I, et al. Molecular genetic alterations in endometrioid carcinomas of the ovary: similar frequency of beta-catenin abnormalities but lower rate of microsatellite instability and PTEN alterations than in uterine endometrioid carcinomas. Hum Pathol 2004;35:1360-1368.

42 Oliva E, Sarrio D, Brachtel EF, et al. High frequency of beta-catenin mutations in borderline endometrioid tumours of the ovary. J Pathol 2006;208:708-713.

$43 \mathrm{Wu}$ R, Zhai Y, Fearon ER, et al. Diverse mechanisms of beta-catenin deregulation in ovarian endometrioid adenocarcinomas. Cancer Res 2001;61:8247-8255.

44 Wang Y, Helland A, Holm R, et al. PIK3CA mutations in advanced ovarian carcinomas. Hum Mutat $2005 ; 25: 322$.

45 Flesken-Nikitin A, Choi KC, Eng JP, et al. Induction of carcinogenesis by concurrent inactivation of p53 and Rb1 in the mouse ovarian surface epithelium. Cancer Res 2003;63:3459-3463. 